\title{
Ischemic Colitis
}

\author{
Mark Y. Sun, M.D. ${ }^{1}$ and Justin A. Maykel, M.D. ${ }^{1}$
}

Ischemic colitis is the most common form of gastrointestinal ischemia. Patients present with either occlusive or nonocclusive vascular disease, although the latter is more common. Many causes of nonocclusive disease have been identified, but the exact pathophysiology remains unclear. Most commonly, patients develop abdominal discomfort and bloody diarrhea. Diagnosis is confirmed with colonoscopy. Treatment is contingent on the severity of disease: mucosal/nongangrenous ischemia requires only supportive measures and medical management, whereas transmural/gangrenous ischemia may require prompt surgical intervention. Ischemic colitis can also become a chronic process with persistent segmental colitis or colonic stricturing. The patient's outcome depends on the severity of disease, prompt recognition, and appropriate treatment.

KEYWORDS: Ischemic colitis, colonoscopy, gastrointestinal bleeding, colectomy, proctosigmoiditis

Objectives: On completion of this article, the reader should be able to summarize the management of ischemic colitis.

Ischemic colitis is the most common form of gastrointestinal ischemia, accounting for $50 \%$ to $60 \%$ of all cases and roughly 1 in 2000 hospital admissions. ${ }^{1}$ The causes of ischemic colitis are numerous, but all result in an inadequate blood supply to the colon, leading to either mucosal or full-thickness injury. The clinical consequences range from being self-limited to catastrophic.

Gangrene of the colon has been recognized for well over a century, but it was not until the 1950s that the full spectrum of ischemic manifestations became apparent. These phenomena were first described following high ligation of the inferior mesenteric artery during abdominal aortic aneurysm repair and colectomy. ${ }^{2,3}$ Following a series of clinical and experimental animal studies in 1963, Boley et al were the first to describe noniatrogenic, reversible colonic injury. ${ }^{4}$ In a follow-up study in 1965, the same group outlined the late consequences of noniatrogenic ischemia including stricture, gangrene, and chronic colitis. ${ }^{5}$ In 1966, Marston et al coined the term "ischemic colitis" to describe the assortment of previously described ischemic injuries. ${ }^{6}$

Unfortunately, the diagnosis of ischemic colitis remains a challenge, as the symptoms are often selflimited and nonspecific. The exact etiology of the initial insult is rarely elucidated, particularly as this disease is most commonly seen in elderly, debilitated patients. Other diseases such as inflammatory and infectious colitis manifest similarly, making the true incidence of ischemic colitis unknown. Nevertheless, the patient's outcome rests on the severity, extent, and rapidity of the ischemic insult and is strongly influenced by prompt diagnosis and appropriate clinical management.

\section{VASCULAR ANATOMY}

To understand the pathophysiology and etiology of ischemic colitis, we must first understand the vascular
${ }^{1}$ Department of Surgery, Section of Colon and Rectal Surgery, University of Massachusetts Medical School, Worcester, Massachusetts.

Address for correspondence and reprint requests: Justin A. Maykel, M.D., Department of Surgery, Section of Colon and Rectal Surgery, University of Massachusetts Medical School, 67
Belmont St., Worcester, MA 01605. E-mail: maykelj@ummhc.org. Miscellaneous Colitides; Guest Editor, Judith L. Trudel, M.D. Clin Colon Rectal Surg 2007;20:5-12. Copyright (C) 2007 by Thieme Medical Publishers, Inc., 333 Seventh Avenue, New York, NY 10001, USA. Tel: +1(212) 584-4662.

DOI 10.1055/s-2007-970194. ISSN 1531-0043. 
anatomy of the colon. The colon and rectum derive their blood supply from branches of three main vessels: the superior mesenteric artery (SMA), the inferior mesenteric artery (IMA), and the paired internal iliac arteries (Fig. 1).

The SMA arises directly from the aorta and supplies both the ascending colon and the proximal two thirds of the transverse colon through the ileocolic, right colic, and middle colic branches. Anatomic variants of these vessels include an absent right colic artery in $2 \%$ to $18 \%$ of patients and an absent middle colic artery in up to $20 \%$ of patients. ${ }^{8,9}$ The IMA arises from the distal abdominal aorta. It gives rise to the left colic artery, which supplies the distal one third of the transverse colon and the proximal portion of the descending colon, and the sigmoid branches, which supply the remainder of the colon. The descending branch of the IMA ends as the superior rectal (hemorrhoidal) artery, which is the principal blood supply to the upper and middle rectum. In addition, the middle and lower rectum receives circulation through the paired middle and inferior hemorrhoidal arteries, coursing from the internal iliac arteries.

Almost as important as the primary circulation to the colon is the expansive collateral circulation. The marginal artery of Drummond parallels the wall of the entire colon and gives rise to the vasa recta. It receives input from the terminal portions of each colic artery. The primary connection between the superior and IMA systems occurs via the left branch of the middle colic artery and the ascending branch of the left colic artery.
However, as many as $30 \%$ of patients have underdeveloped or absent connections between these two arterial systems in the region of the splenic flexure, which makes this area susceptible to ischemic injury. ${ }^{10}$ This region of poor collateral circulation is often referred to as a "watershed" area and has been coined "Griffith's point." In addition, the region between the last sigmoid branch and the superior hemorrhoidal artery may be susceptible to ischemic injury and has been referred to as "Sudeck's point." The meandering mesenteric artery, although not always present, can represent another potential connection between the SMA and IMA systems. This is a large, tortuous vessel that can become particularly prominent in patients with intrinsic mesenteric stenotic disease. The IMA and internal iliac arteries communicate through the superior and middle hemorrhoidal arteries.

\section{ETIOLOGY AND PATHOPHYSIOLOGY}

Colonic blood flow can be compromised by either changes in the entire systemic circulation or alterations within the local mesenteric vasculature. The causes of ischemic colitis appear countless (Table 1). Occasionally a definite cause of ischemia can be recognized, but in most cases none is identified. The greater incidence of ischemic events in older patients suggests a relation to degenerative changes in the vascular tree, but abnormalities on angiography have rarely correlated with clinical disease. ${ }^{1}$ This finding may be in part due to limitations of angiography when evaluating smaller vessels. ${ }^{1}$

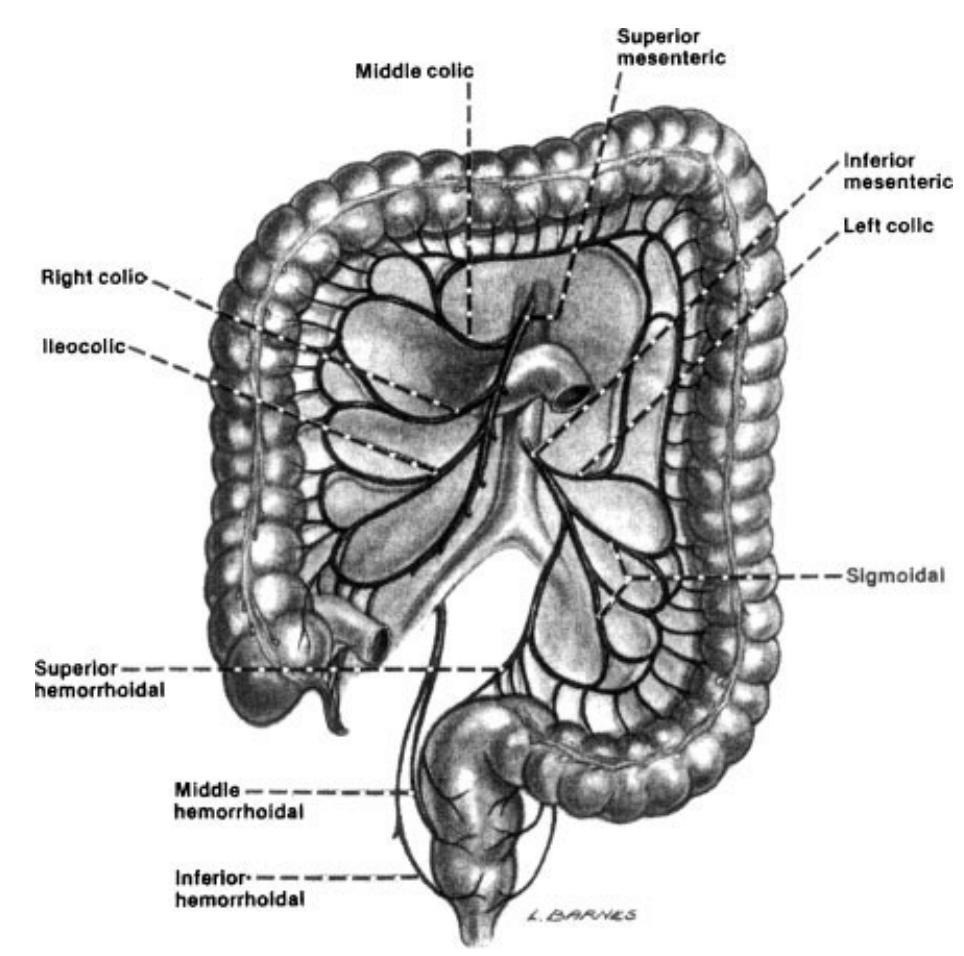

Figure 1 Vascular supply of the colon. Reproduced with permission from Corman ML. Anatomy of the Colon. In: Corman LL, ed. Colon and Rectal Surgery. 5th edition. Philadelphia, PA: Lippincott Williams \& Wilkins; 2005: 1-29. 
Table 1 Causes of Ischemic Colitis

\begin{tabular}{ll}
\hline Occlusive Causes & Nonocclusive Causes \\
\hline Arterial & Idiopathic \\
Arterial embolus & Shock \\
Cholesterol emboli & Cardiogenic \\
Thrombosis & Hypovolemic \\
Trauma & Hemorrhagic \\
Post-aortic reconstructive surgery & Septic \\
Colectomy with IMA ligation & Allergy \\
Small vessel disease & Colonic obstruction \\
Diabetes & Volvulus \\
Amyloidosis & Colon cancer \\
Vasculitis & Pseudo-obstruction \\
Radiation injury & Strangulated hernia \\
Rheumatoid arthritis & Fecal impaction \\
Venous & Medication \\
Hematologic disorder & Digitalis \\
Protein C and S deficiency & Estrogens \\
Antithrombin III deficiency & Cocaine \\
Sickle-cell disease & NSAIDs \\
Mesenteric venous thrombosis & Diuretics \\
Pancreatitis & Danazol \\
Portal hypertension & Long-distance running \\
\hline
\end{tabular}

IMA, inferior mesenteric artery; NSAID, nonsteroidal anti-inflammatory drug.

Ischemic colitis is commonly divided into two groups based on etiology: occlusive or nonocclusive. Occlusive ischemia can result from either arterial inflow or venous outflow obstruction, although the latter is rare. ${ }^{10}$ Arterial obstruction is most commonly caused by embolus or thrombosis but occasionally can be due to other factors such as trauma or surgery. ${ }^{10}$ Nonocclusive ischemia is now a well-recognized condition. "Spontaneous" episodes of ischemia are generally viewed as localized forms of nonocclusive ischemia. ${ }^{1}$ The precipitating event is commonly related to conditions that cause hypotension or shock such as cardiac failure, hemorrhage, or sepsis. Various medications as well as colonic obstruction have also been implicated in the pathogenesis of disease. Patients with nonocclusive ischemia may have preexisting vascular abnormalities, but it is a triggering event that typically leads to a lowflow state or an intense vasoconstriction with shunting of the mesenteric circulation. ${ }^{11}$

It remains unknown whether the colon's susceptibility to ischemic injury is due to (1) an increased demand by the colonic tissue superimposed on an already marginal circulation or (2) an acutely diminished flow to the colon. Regardless of which it is, several factors are thought to predispose the colon to ischemic injury. First, the colon receives less blood flow than any other portion of the gastrointestinal (GI) tract. ${ }^{12}$ Second, the colon's blood flow decreases during periods of functional motor activity (as opposed to the small intestine, where blood flow increases with digestion and peristalsis). Finally, the blood flow to the colon appears to be strongly affected by autonomic stimulation, as found in animal experiments. ${ }^{13}$

\section{CLINICAL PRESENTATION}

Despite variable causes, patients with colonic ischemia tend to present similarly. Patients typically complain of the sudden onset of crampy, mild abdominal pain. In fact, abdominal pain is one of the most commonly associated symptoms and is present in two thirds of all patients. ${ }^{11}$ Although generally poorly localized, ischemia arising in the left colon and rectum tends to be referred to the left lower quadrant and flank area and ischemia arising in the transverse and right colon refers symptoms to the central abdomen. ${ }^{10}$ Sometimes these physical finding can be a clue to etiology, as systemic low-flow states have a propensity to affect the right side of the colon, whereas localized nonocclusive ischemic injury tends to affect the watershed areas of the colon such as the splenic flexure and junction of the sigmoid and rectum. ${ }^{1}$ Abdominal pain is soon followed by an urge to defecate and subsequent passage of either bright red blood or maroon blood mixed with stool, typically within 24 hours of onset. Bleeding is usually insignificant in quantity, and blood loss requiring transfusion is unusual and should prompt the search for an alternative diagnosis. ${ }^{1}$ Nausea, vomiting, abdominal distention, anorexia, and ileus are less common but may also be present.

Physical examination often reveals only mild to moderate tenderness over the involved segments of colon. Of course, when patients develop transmural ischemia, the parietal peritoneum becomes involved and the patient often develops peritoneal signs such as rebound and guarding. Such peritoneal signs vary from localized to diffuse, depending on the extent of colonic injury. The majority of patients, however, present without signs of peritonitis or hemodynamic instability, as most do not have transmural disease.

The differential diagnosis of ischemic colitis is multiple and includes, but is not limited to, mesenteric artery insufficiency, mesenteric venous thrombosis, inflammatory bowel disease, infectious colitis, pseudomembranous colitis, diverticulitis, peptic ulcer disease, bowel obstruction, and pancreatitis.

\section{DIAGNOSIS}

The diagnosis of ischemic colitis is often made by the combination of early and repeated clinical evaluations, radiologic studies, and colonoscopic visualization. Work-up begins with a proper history and physical examination. The clinical setting in which symptoms 
occur is an important clue and piques the evaluator's level of suspicion. For example, ischemic colitis in younger patients may develop following the use of certain medications, marathon running, or profound hypotensive episodes. On the other hand, ischemic colitis occurs in the older population in the presence of atherosclerotic vascular disease, congestive heart failure, and coronary artery disease.

Before proceeding with the work-up for ischemic colitis, however, mesenteric ischemia must be excluded. Occasionally, the diagnosis of mesenteric ischemia is entertained because (1) only the right side of the colon is affected (within the distribution of the SMA) or (2) the patient has more severe abdominal pain or physical findings than are expected with colonic ischemia alone. Although angiography is not indicated for the diagnosis of ischemic colitis, it may be extremely helpful in the management of mesenteric ischemia, from both a diagnostic and therapeutic standpoint. In general, the diagnosis of mesenteric ischemia should be ruled out prior to barium or other contrast-related studies, as the administration of such compounds interferes with adequate angiographic imaging. In this case, an air enema can help to differentiate between mesenteric and colonic ischemia if the remainder of the clinical presentation is unclear. The submucosal edema and hemorrhage that produce the thumbrinting pattern of colonic ischemia can be demonstrated against the column of air. It is important not to overdistend the colon, however, as this may cause hemodynamic instability or loss of the thumbprints, or both. ${ }^{1}$ When a provisional diagnosis of ischemic colitis has been made, it is appropriate to proceed with laboratory tests, further radiologic studies, and possible endoscopic evaluation.

\section{Laboratory Tests}

Laboratory values are variable and nonspecific but most often reveal a mild leukocytosis with a left shift. Occasionally, electrolyte and renal abnormalities may be present secondary to dehydration or even a slight metabolic acidosis. The lactate level may be elevated, but this is the case with any tissue hypoxia and is therefore not a specific marker. Unfortunately, no biochemical marker exists to date that is reliable in identifying colonic ischemia. Reports of measuring serum levels of D-lactate (a stereoisomer of lactate) have shown some promise. D-Lactate is produced by bacteria, particularly within the colon. It is strictly a product of bacterial fermentation, and in healthy individuals the serum levels are negligible. ${ }^{14} \mathrm{With}^{\mathrm{co}}$ lonic ischemic injury, mucosal permeability increases, allowing D-lactate to enter the portal circulation. Because the liver is unable to metabolize D-lactate, levels measured in the systemic circulation closely mimic those in the portal circulation. D-Lactate was found in two studies to have a sensitivity of $82 \%$ to $90 \%$ and a specificity of $77 \%$ to $87 \%$ in predicting early colonic ischemia. ${ }^{14,15}$

\section{Radiologic Studies}

Plain radiographs have limited use in the diagnosis of colonic ischemia. In $\sim 20 \%$ of patients, specific signs of colonic ischemia may be present, including thumbprinting from mural thickening and rarely pneumatosis coli, a sign of advanced disease. ${ }^{16}$ However, the majority of films are nonspecific, showing only dilation or air-fluid levels, or both. The main utility in obtaining plain films is to rule out visceral perforation and the presence of pneumoperitoneum.

Computed tomography (CT) imaging is now widely available and typically used as the first study in assessing the patient with abdominal pain. It can be a very useful test in ruling out other conditions that may arise similarly to ischemic colitis, but its effectiveness in diagnosing colonic ischemia remains marginal. It may provide indirect evidence of ischemic colitis, such as mural thickening or ascites, but the gross morphologic features overlap with those of other colitides and limit its specificity. ${ }^{17}$ More important, CT findings have not correlated well with, nor have they predicted, the development of bowel infarction. ${ }^{17}$

Findings on air contrast or barium/air contrast enemas can be predictive of ischemic colitis, but often they do not exclude other forms of colitis such as inflammatory bowel disease or pseudomembranous colitis. ${ }^{10}$ Thumbprinting or pseudotumors are indicative of submucosal hemorrhage and edema-both of which are found in ischemic colitis. Because neither barium, water-soluble, nor air contrast enemas can provide a conclusive diagnosis of ischemic colitis, their use in the acute setting is limited. In addition, the risk of worsening the ischemic process with overdistention of the colon provides further reason to use these studies with caution. Barium enema, however, is still a useful tool when employed for evaluation or surveillance of chronic problems associated with ischemic colitis such as stricture or persistent colitis. ${ }^{11}$

Color Doppler and duplex ultrasound studies have also been helpful in distinguishing between inflammatory bowel disease and colonic ischemia. Teefey et al evaluated 35 patients with either inflammatory or ischemic bowel wall thickening by both duplex and color Doppler ultrasonography. They found that ultrasonography can help differentiate between the two disease processes. When an arterial signal or color Doppler flow is not detected, ischemia should be a strong consideration and inflammation virtually excluded. However, only $50 \%$ of patients with ischemia demonstrated this finding. ${ }^{18}$ Although ultrasonography has demonstrated 


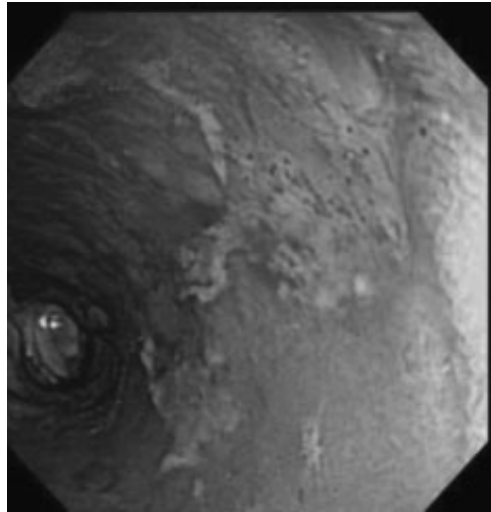

Figure 2 Colonoscopy-early colonic ischemia.

adequate specificity, the sensitivity does not allow it to be a reliable primary diagnostic tool.

\section{Endoscopy}

Colonoscopy remains the most sensitive and specific study available for diagnosis of ischemic colitis. In early ischemia, the mucosa appears pale and edematous with interspersed areas of hyperemia (Fig. 2). ${ }^{19}$ As the ischemic process progresses, evidence of submucosal edema and hemorrhage can be seen as bluish-black blebs or nodules protruding into the lumen of the bowel (Fig. 3).$^{10}$ These lesions create the characteristic thumbprinting sign seen on radiographic studies. The initial diagnostic study should be obtained early in the course because thumbprinting disappears within days as the submucosal hemorrhages are either resorbed or evacuated into the colon when the overlying mucosa ulcerates and sloughs. ${ }^{1}$

In addition to visual inspection, colonoscopy allows the examiner to sample the colonic mucosa for pathologic assessment. Although visualization of the colon may confirm the diagnosis of colitis, biopsy results can help to differentiate inflammatory, infectious, and ischemic etiologies. The histological evidence of mucosal infarction or the appearance of ghost cells is pathogno-

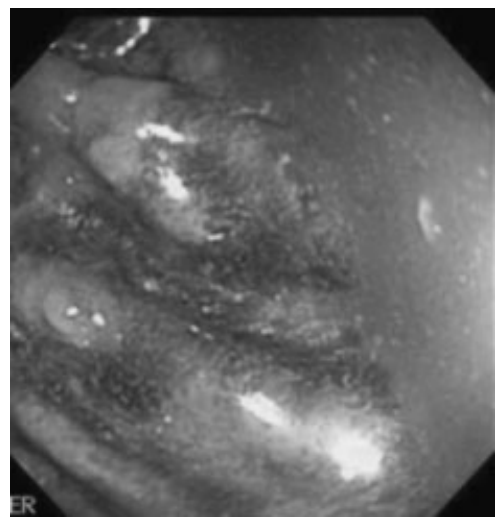

Figure 3 Colonoscopy_-progressing colonic ischemia. monic for ischemia. One limitation of colonoscopy is that only the colonic mucosa can be visualized and biopsied, making it difficult to differentiate mucosal from transmural ischemia. Occasionally, extensive areas of gray-green or black mucosa and submucosa are identified, which can signify transmural infarction, ${ }^{7}$ but this, fortunately, is an uncommon finding.

If colonoscopy is chosen as the initial study, it is important to proceed with caution. Distention of the bowel to pressures greater than $30 \mathrm{~mm} \mathrm{Hg}$ diminishes the colonic blood flow and may actually worsen the colonic ischemia. ${ }^{20}$ This risk can be minimized by insufflating with $\mathrm{CO}_{2}$ rather than room air because $\mathrm{CO}_{2}$ is rapidly absorbed from the intestine and also has the unique property of acting as a vasodilator, potentially increasing colonic blood flow. ${ }^{20}$ In addition, the ischemic wall of the colon is weak and at risk of perforating with the application of even minimal force, so any examination should proceed with great vigilance.

\section{MANAGEMENT}

When a preliminary diagnosis of ischemic colitis is made and the patient does not show signs of intestinal gangrene or perforation, it is generally appropriate to manage the patient nonsurgically. Because most patients present with only mucosal ischemia, their clinical course is often relatively benign. Intravenous fluids should be started and the patient placed at bowel rest. Cardiac function and oxygen delivery should be optimized and, if possible, any medications that are known to cause mesenteric vasoconstriction should be discontinued. If an ileus is present or the colon is markedly dilated, a nasogastric and/or rectal tube should be placed, although one should use a rectal tube in this setting with great caution. In most institutions, patients are also administered broad-spectrum antibiotics to cover both aerobic and anaerobic coliform bacteria. Although there is no good evidence that antibiotics alter the course of disease, they are thought to be efficacious for two reasons: (1) colonic ischemia predisposes to mucosal permeability and the potential for bacterial translocation, and (2) animal studies have shown that antibiotics can reduce the length and severity of bowel damage. ${ }^{21,22}$ The use of pharmacologic intervention, such as glucagon and papaverine, has been described to increase intestinal circulation, but the benefit has not been well documented and therefore this practice has yet to gain wide clinical use. ${ }^{23}$ No attempt should be made to prepare the bowel for surgery during the acute phase of the illness as this may precipitate a colonic perforation or toxic dilation. ${ }^{1}$

Patients should continue to be monitored closely for signs of clinical deterioration, which would include rising temperature, increasing abdominal tenderness, guarding, worsening leukocytosis, sepsis, or free air. If 
colonic infarction is suspected, a prompt exploratory laparotomy and resection are warranted. At the time of surgery, the margins of resection should be dictated by the distribution of disease. The serosa can often appear normal even when the underlying mucosa is affected, which is why visual inspection of the colon alone can be inadequate. Intraoperative colonoscopy can aid in the decision prior to resection but can often be a technically challenging endeavor. Another option is simply opening the resected specimen in the operating room for direct mucosal inspection. Either way, healthy mucosa must be seen at the resection margins to ensure removal of all diseased bowel. In general, by the time the decision has been made to proceed with surgery, the ischemic insult is both diffuse and advanced. Therefore, the preferred option remains a total abdominal colectomy with end ileostomy. Primary anastomosis after a subtotal colectomy or partial colectomy is generally a poor option; it may be considered only when the patient is hemodynamically stable, the bowel margins are clearly viable, and the anastomosis is protected with a diverting upstream stoma (preferably a loop ileostomy). ${ }^{10}$ Regardless of the approach, patients treated operatively have a mortality as high as $40 \%$, particularly when they have other diagnoses such as atherosclerosis or chronic renal failure. ${ }^{24}$

\section{PROGNOSIS}

In most cases of ischemic colitis, the signs and symptoms of disease disappear within 24 to 48 hours. The submucosal and intramural hemorrhages are reabsorbed, and complete clinical and radiologic resolution occurs within 1 to 2 weeks, after which time no further therapy is required. ${ }^{1}$ More severe initial ischemic insults may result in necrosis of the overlying mucosa, resulting in a subsequent segmental ulcerating colitis. Patients with asymptomatic segmental colitis may go on to develop a stenosis or stricture of the colon. Strictures that are not clinically significant can merely be observed, and some of these may resolve on their own in 12 to 24 months with no therapy. ${ }^{1}$ However, a follow-up colonoscopy is advisable to confirm complete resolution or to document the development of a stricture or persistent colitis, or both. Approximately $10 \%$ of patients who initially recover from their ischemic event may present several months later with obstructive symptoms caused by a stricture. ${ }^{10}$ Diagnosis of a stricture is confirmed by colonoscopy or barium enema, and surgical resection is recommended. Typically, these operations proceed on an elective basis, allowing segmental resection and primary anastomosis. In addition, another $20 \%$ to $30 \%$ of patients go on to develop chronic colitis from irreversible ischemic injury, ${ }^{25}$ manifested by persistent diarrhea, rectal bleeding, and/or weight loss. If suspected, this too is likely to require surgical intervention. Recurrent episodes of sepsis in otherwise asymptomatic patients thought to be a result of unhealed areas of segmental colitis should also be considered for elective resection. ${ }^{1}$

\section{SPECIAL TOPICS}

\section{Ischemic Colitis following Aortic Reconstruction}

Ischemic colitis is a well-known complication following aortic surgery. In retrospective studies, the reported incidence of colonic ischemia is $1 \%$ to $3 \%$, increasing to as much as $27 \%$ for patients operated on for a ruptured abdominal aortic aneurysm (AAA). ${ }^{26-32}$ These reports come from highly specialized centers with a small proportion of emergency cases or from small case series. A Norwegian multicenter study including 444 patients operated on for AAA reported a $2 \%$ risk for intestinal ischemia after elective surgery and a $10 \%$ risk after rupture. ${ }^{33}$ Numerous factors can contribute to postoperative colonic ischemia including hemodynamic instability, hypoxemia, prolonged aortic cross-clamp time, dysrhythmias, operative trauma to the colon, improper management of the IMA, and aneurysmal rupture.

Management of ischemic colitis following aortic reconstruction begins with prevention. Preoperative CT arteriography or aortography with selective mesenteric angiography can help elucidate the patency of the celiac axis, SMA, IMA, internal iliac arteries, and the interwoven collateral system. An intact collateral system can help to minimize postoperative ischemic damage. Intraoperative ligation of the IMA can result in ischemic colitis if the collateral system is not sufficient to supply circulation to the left side of the colon. Reimplantation of the IMA or revascularization of the SMA or both are required when the SMA is occluded or tightly stenosed because this indicates that the IMA is the primary source of inflow to the small and large intestine. Intraoperatively, cross-clamp time should be minimized and hypotension avoided. There are various methods to assess adequacy of blood flow during surgery, such as Doppler ultrasonography, intravenous fluorescein, measurement of IMA stump pressures, and measurement of intramural colonic $\mathrm{pH}$. However, none of these methods has gained universal acceptance as a result of their individual inadequacies. $7,32,34$

Grossly bloody or guaiac-positive diarrhea is the most common early manifestation of ischemic colitis and usually occurs within 1 to 2 days of aortic reconstruction. Metabolic acidosis, leukocytosis, progressive oliguria, tachycardia, and hypotension can develop with aggressive forms of ischemic colitis. ${ }^{7}$ If ischemic colitis is suspected, a colonoscopy should be performed. If ischemia is confirmed, supportive measures including fluid resuscitation and broad-spectrum antibiotics should be started immediately. Adequacy of treatment should be 
monitored with serial endoscopy, and any clinical deterioration mandates reoperation. All portions of ischemic colon should be resected and a stoma created. Primary anastomosis is contraindicated because of risk of anastomotic leak and contamination of the aortic prosthesis.

\section{Ischemic Colitis following Angiographic Embolization for Gastrointestinal Bleeding}

Acute lower GI bleeding can be a potentially life-threatening condition. Although the vast majority of cases stop spontaneously, $\sim 10 \%$ to $15 \%$ of cases require angiographic, endoscopic, or surgical intervention. ${ }^{35}$ Angiography and embolization are being used more frequently as the primary treatment modality for massive lower GI bleeding. Early cases of postembolic transmural colon ischemia with perforation were reported in the 1980s and were thought to be a result of the relative lack of collateral vessels in the lower GI tract compared with the upper GI tract. With newer technologies including microcoils and focused superselective embolization, this complication is rarely seen today. Kuo et al performed an institutional retrospective review as well as a literature survey of all microcoil embolizations for lower GI hemorrhage over a 10-year period from 1992 to 2002. They found that although the incidence of postembolization colonic infarction was estimated as $10 \%$ to $20 \%$ before 1992, there were no reported cases after 1992. The incidence of minor ischemia (conditions resulting in no sequelae and requiring nominal therapy or no treatment) was $21 \%$, and the incidence of major ischemic events (defined as bowel infarction requiring surgery) was $0 \% .{ }^{35}$ Although the total number of cases reported in the literature is small, it appears that superselective embolization is safe with regard to postembolization colonic ischemia.

\section{Ischemic Proctosigmoiditis}

Ischemic proctosigmoiditis is a rare condition, affecting $\sim 3 \%$ of patients with colorectal ischemia. ${ }^{36}$ Rectal ischemia is rare because of the excellent collateral supply. It usually occurs in conjunction with more proximal colonic involvement, but it may occur alone. Bharucha et al postulated that isolated rectosigmoid involvement results from a hemodynamic disturbance superimposed on atherosclerotic narrowing of the aortoiliac vessels. ${ }^{36} \mathrm{It}$ has a very similar presentation to more proximal ischemic colitis as patients often have abdominal or pelvic pain, diarrhea, and GI bleeding. Diagnosis can be aided with the use of rectal biopsy. Most cases can be managed conservatively, as with ischemic colitis. It is important to differentiate isolated ischemic proctosigmoiditis from inflammatory bowel disease, as the use of steroids may adversely affect patients with ischemic proctosigmoiditis. Paneth cell metaplasia together with relatively dense inflammation in the lamina propria, as seen on histology, should allow a reliable distinction between the two disease processes. ${ }^{36}$

\section{REFERENCES}

1. Brandt LJ, Boley SJ. Colonic ischemia. Surg Clin North Am 1992;72:203-229

2. Shaw RS, Green TH. Massive mesenteric infarction following inferior mesenteric-artery ligation in resection of the colon for carcinoma. N Engl J Med 1953;248:890-891

3. Smith RF, Szilagyi DE. Ischemia of the colon as a complication in the surgery of the abdominal aorta. Arch Surg 1960;80:806-821

4. Boley SJ, Schwartz S, Lash J, Sternhill V. Reversible vascular occlusion of the colon. Surg Gynecol Obstet 1963;116:53-60

5. Boley SJ, Krieger H, Schultz L, et al. Experimental aspects of peripheral vascular occlusions of the intestine. Surg Gynecol Obstet 1965;121:789-794

6. Marston A, Pheils MT, Thomas ML, Morson BC. Ischemic colitis. Gut 1966;7:1-15

7. Gandhi SK, Hanson MM, Vernava AM, Kaminski DL, Longo WE. Ischemic colitis. Dis Colon Rectum 1996;39:88100

8. Michels NA, Siddharth P, Kornblith PL, Parke WW. The variant blood supply to the descending, rectosigmoid, and rectum based on 400 dissections. Its importance in regional resections: a review of the literature. Dis Colon Rectum $1965 ; 8: 251-278$

9. Griffiths JD. Surgical anatomy of the blood supply of the distal colon. Ann R Coll Surg Engl 1956;19:241-256

10. Macdonald PH. Ischaemic colitis. Best Pract Res Clin Gastroenterol 2002;16:51-61

11. Bower TC. Ischemic colitis. Surg Clin North Am 1993;73: 1037-1053

12. Geber WF. Quantitative measurements of blood flow in various areas of the small and large bowel. Am J Physiol 1960;198:985-986

13. Gilsdorf RB, Urdenata LF, Leonard AS, et al. Posterior hypothalamic effect on gastrointestinal blood flow in the conscious cat. Proc Soc Exp Biol Med 1973;143:329-334

14. Murray MJ, Gonze MD, Nowak LR, Cobb CF. Serum Dlactate levels as an aid to diagnosing acute intestinal ischemia. Am J Surg 1994;167:575-578

15. Poeze M, Froon AHM, Greve JWM, Ramsay G. D-Lactate as an early marker of intestinal ischemia after ruptured abdominal aortic aneurysm repair. Br J Surg 1998;85:12211224

16. Wolf EL, Sprayregen S, Bakel CW, et al. Radiology in intestinal ischemia: plain film contrast, and other imaging studies. Surg Clin North Am 1992;72:107-124

17. Balthazar EJ, Yen BC, Gordon RB. Ischemic colitis: CT evaluation of 54 cases. Radiology 1999;211:381-388

18. Teefey SA, Roarke MC, Brink JA, et al. Bowel wall thickening: differentiation of inflammation from ischemia with color Doppler and duplex US. Radiology 1996;198:547551

19. Scherpenisse J, van Hees PAM. The endoscopic spectrum of colonic mucosal injury following aortic aneurysm resection. Endoscopy 1989;21:174-176

20. Brandt LJ, Boley SJ, Sammartano RJ. Carbon dioxide and room air insufflation of the colon: effects on colonic blood 
flow and intraluminal pressure in the dog. Gastrointest Endosc 1986;32:324-329

21. Path EJ, McClure JN. Intestinal obstruction: protective action of sulfasuxidine and sulfathalidine to ileum following vascular damage. Ann Surg 1950;131:159-163

22. Sarnoff SJ, Fine J. Effect of chemotherapy on ileum subjected to vascular injury. Ann Surg 1945;121:74-78

23. Fitzgerald SF, Kaminski DL. Ischemic colitis. Semin Colon Rectal Surg 1993;4:222-228

24. Scharff JR, Longo WE, Vartanian SM, et al. Ischemic colitis: spectrum of disease and outcome. Surgery 2003;134:624-630

25. Cappell MS. Intestinal (mesenteric) vasculopathy III. Ischemic colitis and chronic mesenteric ischemia. Gastroenterol Clin North Am 1998;27:827-860

26. Zelenock GB, Stordel WE, Knol JA, et al. A prospective study of clinically and endoscopically documented colonic ischemia in 100 patients undergoing aortic reconstructive and aggressive colonic and direct pelvic revascularization, compared with historic controls. Surgery 1989;106:771-779

27. Hagihara PF, Ernst CB, Griffen WB. Incidence of ischemic colitis following abdominal aortic reconstruction. Surg Gynecol Obstet 1979;149:571-573

28. Brewster DC, Franklin DP, Cambria RP, et al. Intestinal ischemia complicating abdominal aortic surgery. Surgery 1991;109:447-454
29. Welling RE, Roedersheimer LR, Arbaugh JJ, Cranley JJ. Ischemic colitis following repair of ruptured abdominal aortic aneurysm. Arch Surg 1985;120:1368-1370

30. Maupin GE, Rimar SD, Villalba M. Ischemic colitis following abdominal aortic reconstruction for ruptured aneurysm: a 10-year experience. Am Surg 1989;55:378-380

31. Valentine JR, Hagino RT, Jackson MR, et al. Gastrointestinal complications after aortic surgery. J Vasc Surg 1998;28:404411

32. Bjorck M, Troeng T, Bergqvist D. Risk factors for intestinal ischaemia after aortoiliac surgery: a combined cohort and case-control study of 2824 operations. Eur J Vasc Endovasc Surg 1997;13:531-539

33. Amundsen S, Trippestad A, Viste A, Soreide O. Abdominal aortic aneurysms - a national multicentre study. Eur J Vasc Surg 1987;1:239-243

34. Bergman RT, Gloviczki P, Welch TJ, et al. Role of intravenous fluorescein in detection of colon ischemia during aortic reconstruction. Ann Vasc Surg 1992;6:74-79

35. Kuo WT, Lee DE, Saad WEA, et al. Superselective microcoil embolization for the treatment of lower gastrointestinal hemorrhage. J Vasc Interv Radiol 2003;14:1503-1509

36. Bharucha AE, Tremaine WJ, Johnson CD, Batts KP. Ischemic proctosigmoiditis. Am J Gastroenterol 1996;91: 2305-2309 